\title{
Dietary PUFA intakes in children with attention-deficit/hyperactivity disorder symptoms
}

\author{
Ka-Hung $\mathrm{Ng}^{1}$, Barbara J. Meyer ${ }^{1}$, Lauren Reece ${ }^{1}$ and Natalie Sinn $^{2}$ \\ ${ }^{1}$ School of Health Sciences, University of Wollongong, Northfields Avenue, Wollongong, NSW 2522, Australia \\ ${ }^{2}$ Nutritional Physiology Research Centre, School of Health Sciences, University of South Australia, Adelaide, SA 5000, Australia
}

(Received 3 October 2008 - Revised 14 April 2009 - Accepted 8 June 2009 - First published online 27 July 2009)

Research has shown associations between attention-deficit/hyperactivity disorder (ADHD) and erythrocyte long-chain $n$-3 PUFA (LC $n$-3 PUFA) levels, with limited evidence for dietary LC $n$-3 PUFA intake and ADHD. The aims of the present study were to assess dietary PUFA intakes and food sources in children with ADHD, to compare these intakes to previously published Australian National Nutrition Survey (NNS) data and determine any relationships between intakes and ADHD symptoms. Eighty-six 3-d-weighed food records (FR) were analysed from children with ADHD. The median (interquartile range) daily intakes of fatty acids (mg/d) were: linoleic acid (18:2n-6), 7797 (6240-12333); arachidonic acid (20:4n-6), 55 (27.0-93); total $n$-6 PUFA, 7818 (6286-10662); $\alpha$-linolenic acid (18:3n-3), 1039 (779-1461); EPA (20:5n-3), 18 (6.0-32.0); docosapentaenoic acid (22:5n-3), $17(6 \cdot 3-39 \cdot 3)$; DHA (22:6n-3), 16 (8.5-445); total LC $n$-3 PUFA (addition of $20: 5 n-3,22: 5 n-3$ and $22: 6 n-3), 65(28 \cdot 3-120 \cdot 1)$; total $n-3$ PUFA, 1151 (876-1592). In comparison to the NNS data, $18: 3 n-3$ intakes were higher and 20:4n-6 were lower $(P<0 \cdot 05)$. Children with ADHD consumed half the amount of fish/seafood, meat and eggs when compared to the NNS $(P<0 \cdot 05)$. No significant correlations were found between fatty acids and ADHD symptoms. Children with ADHD met the adequate intake for LC $n$-3 PUFA, but fell short of other recommendations.

PUFA: Attention-deficit/hyperactivity disorder: $n$-3: Dietary intake

Attention-deficit/hyperactivity disorder (ADHD) is the most common developmental disorder in childhood ${ }^{(1)}$. The main symptoms include poor impulse control, hyperactivity and inattention $^{(2)}$. These problems can adversely affect school performance, family relationships and social interactions ${ }^{(3,4)}$. Children with ADHD often experience co-morbidity with other behavioural disorders including anxiety, conduct, oppositional and/or mood disorders and antisocial personality ${ }^{(5)}$. The prevalence rates are estimated at $4-15 \%$ in $\mathrm{USA}^{(6)}$ and $11 \%$ in Australia ${ }^{(1)}$. The variation in prevalence is probably due to the differences in diagnostic criteria. Boys are more commonly diagnosed with ADHD than girls ${ }^{(7)}$. It is now clear that problems associated with ADHD also persist into adulthood ${ }^{(8)}$.

The aetiology of ADHD appears to be multifactorial with genetic and environmental influences. A review has supported an association between ADHD and polymorphisms ${ }^{(9)}$. Cigarette smoking during pregnancy has been found to increase the risk of ADHD in children ${ }^{(10)}$. Focus has also been placed on diet, including emerging evidence that ADHD may be associated with low levels of dietary and erythrocyte long-chain $n-3$ PUFA (LC $n-3$ PUFA) ${ }^{(3,4,11)}$. The LC $n-3$ PUFA, including EPA $(20: 5 n-3)$ and DHA $(22: 6 n-3)$, can be converted from $\alpha$-linolenic acid
$(18: 3 n-3)$ by endogenous desaturation and elongation ${ }^{(12)}$; however, only negligible amounts are converted ${ }^{(13,14)}$. LC PUFA are critical in normal brain and nervous system development and function. $22: 6 n-3$ is highly concentrated in brain and retina, while LC $n-6$ arachidonic acid $(20: 4 n-6)$ together with $22: 6 n-3$ plays a major structural role in neuronal membranes ${ }^{(15)}$.

Therefore, there has been growing interest in the role of $n-3$ PUFA in psychiatric illness across the lifespan ${ }^{(15-17)}$. It has been well established that $22: 6 n-3$ is critical for infant brain development, and 22:6n-3 may be associated with enhanced cognitive performance in childhood ${ }^{(18)}$. A number of studies have found lower blood $n-3$ levels in children with ADHD compared with control groups ${ }^{(19,20)}$ and there is evidence for alleviation of ADHD symptoms with $n$-3 PUFA supplementation $^{(4)}$. Results of trials have been conflicting; although the three largest, randomised placebo-controlled studies have reported improvement in ADHD symptoms in children following supplementation with $732 \mathrm{mg} / \mathrm{d}$ of LC $n-3$ PUFA and $60 \mathrm{mg} / \mathrm{d}$ of $\gamma$-linolenic acid $(18: 3 n-6)$ over $24-30$ weeks $^{(21-23)}$.

However, no specific studies to date have investigated dietary intake of $n-3$ PUFA in children with ADHD to investigate whether these children are consuming low levels of LC $n-3$

Abbreviations: ADHD, attention-deficit/hyperactivity disorder; FR, food records; LC $n-3$ PUFA, long-chain $n$-3 PUFA; NNS, National Nutrition Survey; NRV, Nutrient Reference Values.

* Corresponding author: Barbara J. Meyer, fax +6124221 3486, email bmeyer@uow.edu.au 
PUFA in their diet. Therefore, the aims of the present study were to assess dietary PUFA intakes and food sources in children with ADHD, to compare these intakes to the previously published estimates of children's PUFA intake using the data from the Australian National Nutrition Survey $(\mathrm{NNS})^{(24)}$, to compare these intakes to the Nutrient Reference Values (NRV) ${ }^{(25)}$ and other recommendations ${ }^{(26,27)}$, and to determine whether dietary $n-3$ PUFA intakes and ADHD symptoms are related.

\section{Methods \\ Participants}

A total of 182 children with parent-reported ADHD symptoms above the 90th percentile on the 12-item ADHD Index from Conners' Parent Rating Scales ${ }^{(28)}$ were recruited via media releases, newspaper advertisements and school newsletters, in South Australia and took part in an $n-3$ supplementation trial that has been published ${ }^{(21)}$. Children were included if they were 6-13 years old and had ADHD or parent-reported ADHD symptoms, were not taking fish-oil supplements and were not taking stimulant medication. Eighty-six participants consented to the subsequent analysis of the dietary data. The present study focused on the baseline dietary analysis of these children from the previously published trial ${ }^{(21)}$.

The present study was conducted according to the guidelines laid down in the Declaration of Helsinki, and all procedures involving human subjects/patients were approved by the Human Research Ethics Committees of the Commonwealth Scientific and Industrial Research Organisation and University of South Australia, and the South Australian Department of Education and Children's Services and Catholic Education Centre. Written informed consent was obtained from all subjects/patients.

\section{Anthropometric measurements}

During their first clinic visit, height was measured using a portable stadiometer to the nearest $0.1 \mathrm{~cm}$ and weight was measured using standard scales to the nearest $0.1 \mathrm{~kg}$, both with the children barefoot. BMI was calculated by dividing the weight (in kilograms) by the height (in metres) squared.

\section{Attention-deficit/hyperactivity disorder index from Conners' Parent Rating Scales}

The ADHD index from Conners' Parent Rating Scales ${ }^{(28)}$ was used to assess parent ratings of ADHD symptoms; from this, inattention, hyperactivity and total ADHD scores were used for analysis ${ }^{(28)}$. The ADHD index is an abbreviated form of the long rating scales, essentially the key subscale that measures the core ADHD symptoms of inattention and impulsivity/hyperactivity. The index has twelve statements (e.g. 'inattentive, easily distracted', 'fidgets with hands or squirms in seat') that are scored from 0 (not at all) to 3 (very much). The maximum possible score is therefore 36 .

\section{Food records}

Three-day-weighed FR were collected at baseline from the main study referred to earlier ${ }^{(21)}$. Children and their parents/ caregivers were asked to complete the FR on three different days; two non-consecutive weekdays and one weekend day. Children and their parents/caregivers were shown a sample FR that showed them how to fill it in. They were told to record the brand and type of foods, and to estimate the amount of all eaten foods as accurately as possible. The importance of maintaining normal diets was emphasised.

The Australia Standard database within the FoodWorks nutrient analysis software package (version 5 service pack 1 , Xyris software) was used to determine the energy, macronutrients and micronutrients intakes of each child from their FR. If the foods in the FR could not be exactly matched in the FoodWorks database, these foods were substituted by other similar types of foods. For example, roma tomato was substituted with ordinary tomato.

\section{Validation of food records}

Diet reports of poor validity were identified using the Goldberg cut-off ${ }^{(29)}$. The ratio of energy intake to $\mathrm{BMR}^{(30)}$ of each participants was calculated, values equal to or less than 1.06 were classed as under-reporters and hence eliminated from the analysis. A 3-d-weighed FR is long enough to assess PUFA intake as shown by repeat FR assessments by Sullivan et al. ${ }^{(31)}$.

\section{PUFA intakes}

The Royal Melbourne Institute of Technology Australian Fatty Acids database ${ }^{(32)}$ within the FoodWorks nutrient analysis software package was used to analyse the children's PUFA intakes. Fat content of foods was obtained from the Australia Standard database ${ }^{(32)}$. Again, when the foods in the FR could not be matched in the fatty acids' database, they were substituted by similar types of foods, for example muffin was substituted by cake because they have a similar fat content. Some foods that could not be found in database were entered manually as a new food from manufacturers' information, for instance Tip Top ${ }^{\circledR} \mathrm{Up}^{\mathrm{TM}}$ Omega-3 Bread.

If ambiguous quantity of meat in the FR was given, where the precise weight is not recorded, for example 'one piece of chicken breast', a child serving size of meat was used, which is $65 \mathrm{~g}^{(33)}$. If the size of meat was given, for example 'one large piece of chicken breast', the amount used was $65 \mathrm{~g}$ multiplied by 1.5 . If 'one small piece of chicken breast' was given, the amount used was $65 \mathrm{~g}$ divided by $1 \cdot 5$.

The average daily PUFA intakes were calculated by dividing the total PUFA intakes by three (as 3-d-weighed FR were collected). The average daily $18: 2 n-6,20: 4 n-6$, total $n$-6 PUFA, $18: 3 n-3,20: 5 n-3,22: 5 n-3,22: 6 n-3$, total LC $n$-3 PUFA and total $n-3$ PUFA were determined, in addition to the ratio of $n-3$ PUFA to $n-6$ PUFA, $20: 5 n-3$ to $22: 6 n-3,20: 4 n-6$ to $20: 5 n-3, n-3$ PUFA to total PUFA, LC $n-3$ PUFA to PUFA and LC $n-3$ PUFA to $n$-3 PUFA. 
Table 1. Subject characteristics and attention-deficit/hyperactivity disorder (ADHD) symptoms (Mean values and standard deviations with ranges)

\begin{tabular}{|c|c|c|c|c|c|c|c|c|c|}
\hline & \multicolumn{3}{|c|}{ All $(n 79)$} & \multicolumn{3}{|c|}{ Boys ( $n 57)$} & \multicolumn{3}{|c|}{ Girls (n 22) } \\
\hline & Mean & SD & Range & Mean & SD & Range & Mean & $\mathrm{SD}$ & Range \\
\hline Age (years) & $9 \cdot 3$ & $1 \cdot 7$ & $6-13$ & $9 \cdot 3$ & 1.8 & $6-13$ & $9 \cdot 2$ & 1.5 & $6-12$ \\
\hline Height $(\mathrm{cm})$ & 139.8 & $12 \cdot 4$ & $115-174$ & $140 \cdot 7$ & 13.3 & $115-174$ & 137.4 & 9.6 & $122-154$ \\
\hline Weight (kg) & $35 \cdot 5$ & $10 \cdot 9$ & $20-70$ & 36.4 & 11.9 & $20-70$ & 33.4 & $7 \cdot 4$ & $21-54$ \\
\hline BMI $\left(\mathrm{kg} / \mathrm{m}^{2}\right)$ & 17.8 & 3.2 & $12-28$ & 17.9 & 3.3 & $14-28$ & 17.6 & 3.1 & $12-24$ \\
\hline \multicolumn{10}{|l|}{ ADHD symptoms } \\
\hline Inattention & $13 \cdot 9$ & 1.7 & $4-18$ & $14 \cdot 1$ & 3.5 & $4-18$ & 13.5 & 3.4 & $5-18$ \\
\hline Hyperactivity & $11 \cdot 3$ & 4 & $2-18$ & 11.5 & 4.2 & $2-18$ & 10.95 & 3.4 & $4-16$ \\
\hline Total score & $25 \cdot 1$ & $6 \cdot 2$ & $10-36$ & 25.5 & $6 \cdot 5$ & $10-36$ & 24.4 & $5 \cdot 6$ & $13-32$ \\
\hline
\end{tabular}

\section{Statistical analysis}

All variables were tested for normality using the ShapiroWilk test before further analysis. All variables were not normally distributed, except age, height, weight, BMI and total ADHD score.

The Mann-Whitney $U$ test was used to compare difference between PUFA intakes (including ratio of $n-3$ PUFA to $n-6$ PUFA, $n-6$ PUFA to $n-3$ PUFA, $20: 5 n-3$ to $22: 6 n-3$, $20: 4 n-6$ to $20: 5 n-3, n-3$ PUFA to total PUFA, LC $n-3$ PUFA to PUFA and LC $n-3$ PUFA to $n-3$ PUFA) and food sources in children with ADHD and estimation of PUFA intakes and food sources from the $1995 \mathrm{NNS}^{(24)}$.

Non-parametric Spearman's correlation was used to determine the existence of linear relationships between PUFA intakes (including ratio of $n-3$ PUFA to $n$ - 6 PUFA, $n-6$ PUFA to $n-3$ PUFA, $20: 5 n-3$ to $22: 6 n-3,20: 4 n-6$ to $20: 5 n-3, n-3$ PUFA to total PUFA, LC $n-3$ PUFA to PUFA and LC $n$-3 PUFA to $n$-3 PUFA) and food sources (weight of fish/seafood and meat/egg) and ADHD symptoms.

Participants were ranked in ascending order of their PUFA intakes and divided into two groups, where lower PUFA intakes' group refers to participants consumed lowest $50 \%$ PUFA intakes, and higher PUFA intakes' group refers to participants consumed highest 50\% PUFA intakes. The Mann-Whitney $U$ test was used to compare any difference in ADHD scores between the two groups.

Difference between PUFA intakes in children with ADHD and recommended dietary intakes - $\mathrm{NRV}^{(25)}$ was compared using Wilcoxon signed-rank test.

All data were expressed as the means and standard deviations and range and/or median \pm interquartile range. Statistical analysis was performed using SPSS statistical analysis program (version 15.0.0, SPSS Inc., Chicago, IL, USA). Statistical significance was set at $P<0.05$ for all analyses, except for the multiple correlation analyses where the statistical significance was set at $P<0.01$.

\section{Results}

Eighty-six baseline 3-d-weighed FR were available to analyse from the previous study ${ }^{(21)}$, and seven participants were eliminated as under-reporters. One thousand four hundred and eighty dietary data were available from the $1995 \mathrm{NNS}^{(24)}$, 137 participants were eliminated as under-reporters.

Participant characteristics and ADHD symptoms for this group are shown in Table 1. Participant characteristics in the $1995 \mathrm{NNS}^{(24)}$ are shown in Table 2. No significant difference in ADHD symptoms was found between genders $(P>0.05$; Table 1). Age, height, weight and BMI were not significantly different between the children with ADHD and the children from the NNS $(P>0.05$; Tables 1 and 2$)$.

\section{PUFA intakes and comparison to the National Nutrition Survey}

Daily PUFA intakes and comparison to the NNS data ${ }^{(24)}$ are shown in Table 3. Values are expressed as median and interquartile range rather than mean and SD because of the skewed distribution of PUFA intakes, especially LC $n$-3 PUFA.

In comparison of children PUFA intakes with the NNS data, there were significant differences in certain fatty acids. Intakes of $18: 3 n-3(P<0 \cdot 001)$ and hence total $n$-3 PUFA intakes $(P<0.001)$ were significantly higher and $20: 4 n-6$ intakes $(P<0.05)$ were significantly lower in children with ADHD. The ratio of total $n-3$ PUFA to total $n$ - 6 PUFA

Table 2. Subject characteristics in 1995 National Nutrition Survey ${ }^{(24)}$

(Mean values and standard deviations with ranges)

\begin{tabular}{|c|c|c|c|c|c|c|c|c|c|}
\hline & \multicolumn{3}{|c|}{ All ( $n$ 1343) } & \multicolumn{3}{|c|}{ Boys ( $n$ 722) } & \multicolumn{3}{|c|}{ Girls ( $n$ 621) } \\
\hline & Mean & $S D$ & Range & Mean & SD & Range & Mean & SD & Range \\
\hline Age (years) & $9 \cdot 3$ & $2 \cdot 3$ & $6-13$ & 9.4 & $2 \cdot 3$ & $6-13$ & 9.3 & $2 \cdot 3$ & $6-13$ \\
\hline Height $(\mathrm{cm})^{\star}$ & $139 \cdot 3$ & 14.9 & $105-186$ & $139 \cdot 3$ & 14.8 & $107-186$ & $139 \cdot 2$ & $15 \cdot 1$ & $105-173$ \\
\hline Weight (kg) & 38.9 & $12 \cdot 3$ & $16-86$ & $35 \cdot 3$ & 11.9 & $16-86$ & 36.5 & $12 \cdot 7$ & $16-82$ \\
\hline BMI $\left(\mathrm{kg} / \mathrm{m}^{2}\right)^{*}$ & $17 \cdot 9$ & 3 & $10-31$ & $17 \cdot 7$ & $2 \cdot 8$ & $10-30$ & $18 \cdot 2$ & $3 \cdot 1$ & $13-31$ \\
\hline
\end{tabular}

${ }^{*}$ All $(n$ 1342), boys $(n 722)$, girls $(n 620)$. 
Table 3. Daily PUFA intake and comparison to the National Nutrition Survey (NNS) ${ }^{(24)}$ (Median values and interquartile ranges (IQR))

\begin{tabular}{|c|c|c|c|c|c|}
\hline & \multicolumn{5}{|c|}{ Fatty acid intake (mg/d) } \\
\hline & \multicolumn{2}{|c|}{ ADHD group ( $n 79)$} & \multicolumn{2}{|c|}{ NNS $(n$ 1343) } & \multirow[b]{2}{*}{$P$} \\
\hline & Median & IQR & Median & IQR & \\
\hline \multicolumn{6}{|l|}{$n-6$ PUFA } \\
\hline $18: 2 n-6$ & 7797 & $6240-12334$ & 8072 & $5725-11395$ & NS \\
\hline $20: 4 n-6$ & 54.5 & $27 \cdot 0-93 \cdot 0$ & $74 \cdot 2$ & $35 \cdot 9-134 \cdot 1$ & 0.013 \\
\hline$\Sigma n-6$ PUFA & 7818 & $6286-10662$ & 8252 & $5832-11594$ & NS \\
\hline \multicolumn{6}{|l|}{$n-3$ PUFA } \\
\hline $18: 3 n-3$ & 1039 & $779-1461$ & 787 & $548-1143$ & 0.000 \\
\hline $20: 5 n-3$ & 17.9 & $6 \cdot 0-32 \cdot 0$ & $18 \cdot 7$ & $8 \cdot 1-39 \cdot 8$ & NS \\
\hline $22: 5 n-3$ & $17 \cdot 1$ & $6 \cdot 3-39 \cdot 3$ & $27 \cdot 4$ & $7 \cdot 2-57 \cdot 8$ & NS \\
\hline $22: 6 n-3$ & $15 \cdot 9$ & $8.5-44.5$ & 14.7 & $5 \cdot 1-35 \cdot 8$ & NS \\
\hline LC $n-3$ PUFA & $65 \cdot 2$ & $28 \cdot 3-120 \cdot 2$ & $69 \cdot 2$ & $29 \cdot 7-137 \cdot 7$ & NS \\
\hline$\Sigma n-3$ PUFA & 1151 & $876-1592$ & 883 & $628-1336$ & 0.000 \\
\hline$\Sigma n-3$ PUFA : $\Sigma n-6$ PUFA & 0.14 & $0.12-0.19$ & 0.11 & $0.08-0.15$ & 0.000 \\
\hline$\Sigma n-6$ PUFA : $\Sigma n-3$ PUFA & $7 \cdot 27$ & $5 \cdot 14-8.69$ & 8.82 & $6.46-12.47$ & 0.000 \\
\hline $20: 5 n-3: 22: 6 n-3^{\star}$ & 0.78 & $0.41-1.54$ & 1.00 & $0.58-2.16$ & 0.014 \\
\hline $20: 4 n-6: 20: 5 n-3 \dagger$ & $3 \cdot 18$ & $1.75-5.78$ & $3 \cdot 70$ & $2.29-5.59$ & NS \\
\hline 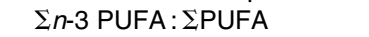 & 0.12 & $0.10-0.16$ & $0 \cdot 10$ & $0.07-0.13$ & 0.000 \\
\hline$\Sigma L C n-3$ PUFA : $\Sigma$ PUFA & 0.006 & $0.003-0.012$ & 0.008 & $0.003-0.015$ & NS \\
\hline$\Sigma$ LC $n-3$ PUFA : $\Sigma n-3$ PUFA & 0.060 & $0.023-0.100$ & 0.079 & $0.033-0.149$ & 0.006 \\
\hline
\end{tabular}

$(P<0 \cdot 001)$ and total $n-3$ PUFA to total PUFA $(P<0 \cdot 001)$ was significantly higher than the NNS, while the ratio of $20: 5 n-3$ to $22: 6 n-3 \quad(P<0 \cdot 05)$, total $n-6$ PUFA to total $n-3$ PUFA $(P<0.001)$ and total LC $n-3$ PUFA to total $n$-3 PUFA $(P<0 \cdot 01)$ was significantly lower compared to the NNS.

There were no significant differences in total energy intake between the children from the NNS and the children with ADHD, and hence expressing the fatty acid intakes data as $\mathrm{mg} / \mathrm{d} / \mathrm{energy}$ showed no significant difference between these two groups (data not shown).

Fish/seafood and meat/egg consumption and the National Nutrition Survey comparison

The amount of fish/seafood and meat/egg (g/d) consumption with comparison to the $\mathrm{NNS}^{(24)}$ is shown in Table 4. Children with ADHD consumed significantly less fish/seafood $(P<0.05)$ and meat/eggs $(P<0.01)$ compared to the NNS. It is of interest to note that less than $25 \%$ of children in the ADHD group and the NNS had consumed fish and/or seafood.
Correlation between PUFA intakes/food sources and attention-deficit/hyperactivity disorder symptoms

Correlation coefficient for ADHD scores and PUFA intakes/ food sources is shown in Table 5. There were no significant correlations found between any of the fatty acids and ADHD symptoms $(P>0 \cdot 01)$. Likewise, no significant correlations were found between amount of fish/seafood and meat/egg consumption and ADHD symptoms $(P>0 \cdot 01)$.

Comparison of attention-deficit/hyperactivity disorder scores between lower/higher PUFA intakes' groups

No significant differences in ADHD scores were found between any lower/higher PUFA intakes' groups (data not shown).

PUFA Intakes and comparison to the nutrient reference values and other recommendations

Comparison of PUFA intakes with $\mathrm{NRV}^{(25)}$ and other recommendations $^{(26,27)}$ are shown in Table 6. Girls consumed

Table 4. Fish, seafood and meat, egg consumption and the National Nutrition Survey (NNS) ${ }^{(24)}$ comparison (Mean values, medians and interquartile ranges (IQR))

\begin{tabular}{|c|c|c|c|c|c|c|c|}
\hline & \multicolumn{7}{|c|}{ Average daily consumption (g/d) } \\
\hline & \multicolumn{3}{|c|}{ ADHD group $(n 79)$} & \multicolumn{3}{|c|}{ NNS $(n$ 1343) } & \multirow[b]{2}{*}{$P$} \\
\hline & Mean & Median & IQR & Mean & Median & IQR & \\
\hline Meat and egg & 106 & 96 & $63 \cdot 3-139 \cdot 2$ & 191 & 142 & $71 \cdot 5-262 \cdot 0$ & 0.000 \\
\hline Fish and seafood & $7 \cdot 1$ & 0 & $0-0$ & $15 \cdot 8$ & 0 & $0-0$ & 0.021 \\
\hline
\end{tabular}

ADHD, attention-deficit/hyperactivity disorder. 
Table 5. Spearman's correlation coefficient for attention-deficit/hyperactivity disorder scores, PUFA intakes and food sources consumption

\begin{tabular}{|c|c|c|c|}
\hline & Inattention & Hyperactivity & Total score \\
\hline $18: 2 n-6$ & 0.05 & -0.08 & -0.04 \\
\hline $20: 4 n-6$ & -0.01 & -0.10 & -0.07 \\
\hline$\Sigma n-6$ PUFA & 0.05 & -0.08 & -0.04 \\
\hline $18: 3 n-3$ & 0.07 & -0.02 & 0.05 \\
\hline $20: 5 n-3$ & -0.23 & -0.10 & -0.20 \\
\hline $22: 5 n-3$ & 0.02 & 0.02 & -0.03 \\
\hline $22: 6 n-3$ & -0.05 & -0.07 & -0.09 \\
\hline$\Sigma$ LC $n-3$ PUFA & -0.04 & -0.03 & -0.05 \\
\hline$\Sigma n-3$ PUFA & 0.07 & -0.01 & 0.05 \\
\hline$\Sigma n-3$ PUFA : $\Sigma n-6$ PUFA & 0.05 & 0.02 & 0.07 \\
\hline$\sum n-6$ PUFA: $\Sigma n-3$ PUFA & -0.05 & -0.02 & -0.07 \\
\hline $20: 5 n-3: 22: 6 n-3$ & -0.09 & -0.02 & -0.04 \\
\hline $20: 4 n-6: 20: 5 n-3$ & 0.01 & -0.01 & -0.01 \\
\hline$\Sigma n-3$ PUFA: $\Sigma$ PUFA & 0.05 & 0.02 & 0.07 \\
\hline$\Sigma$ LC $n-3$ PUFA : $\Sigma$ PUFA & -0.20 & 0.00 & -0.10 \\
\hline$\Sigma$ LC $n-3$ PUFA : $\Sigma n-3$ PUFA & -0.04 & -0.01 & -0.04 \\
\hline Fish/seafood consumption & -0.10 & -0.11 & -0.15 \\
\hline Meat/egg consumption & 0.00 & -0.07 & -0.03 \\
\hline
\end{tabular}

18:2n-6, linoleic acid; $20: 4 n-6$, arachidonic acid; $18: 3 n-3, \alpha$-linolenic acid; $20: 5 n-3$, EPA; $22: 5 n-3$, docosapentaenoic acid; $22: 6 n-3$, DHA; LC $n-3$, longchain $n$-3 PUFA (sum of $20: 5 n-3,22: 5 n-3$ and $22: 6 n-3$ ). $P<0.01$ was used.

significantly more $18: 3 n-3(P=0 \cdot 007)$ than the NRV. Males consumed significantly less $18: 2 n-6$ than the NRV $(P=0.007)$. No significant difference was found between LC $n$-3 PUFA intake and NRV $(P>0.05)$ of both sex. However, in comparison to other recommendations, the LC $n-3$ PUFA intake in children with ADHD was less than $13 \%$ of those recommended intakes as suggested by the National Heart Foundation $^{(26)}$ and the International Society for the Study of Fatty Acids and Lipids ${ }^{(27)}$ (Table 6).

\section{Discussion}

The present analysis of 3-d-weighed FR collected from seventy-nine children with ADHD symptoms indicated that these children had lower $20: 4 n-6$ intake and higher $18: 3$ $n-3$ intake than the general population of the same age. Two previous studies that assessed the diet of children with ADHD compared to age-matched controls found no differences in PUFA intakes ${ }^{(34,35)}$. A small study ( $n$ 11 ADHD and $n 8$ controls) from Canada showed that children with ADHD consumed more energy and saturated fat compared with children without ADHD $^{(35)}$. However, these authors did not take into account the differences in energy intake. If the PUFA intake was expressed as percentage of energy, the mean intake of $20: 4 n-6$ and $22: 6 n-3$ in children with ADHD would be approximately $40 \%$ less than the control group $^{(35)}$. The other larger study ( $n$ 52) did not find any difference in $18: 2 n-6$ and $18: 3 n-3$ intakes in children with ADHD compared to age-matched controls ${ }^{(34)}$. Again, however, if the PUFA intake is expressed as percentage of energy, it is conceivable that the $18: 3 n-3$ intake would be higher than the control group.

Meat and eggs consumption was significantly lower in children with ADHD compared to the NNS, and this was consistent with lower 20:4n-6 intake because meat is a rich source of $20: 4 n-6^{(36)}$. Children with ADHD also consumed significantly lower amounts of fish and seafood compared with children from the NNS. Fish and seafood are the richest source of LC $n-3$ PUFA $^{(36)}$, but lower consumption in the children with ADHD did not translate to significant differences in LC $n$-3 PUFA intake, even though they tended to be lower. Other biochemical data have shown that children with ADHD have a lower level of $20: 4 n-6$ in plasma ${ }^{(19,37)}$ and in erythrocytes ${ }^{(34)}$, while no differences were found in $18: 3 n-3^{(19,34,37)}$, even though dietary PUFA intake may not correlate with blood PUFA ${ }^{(35)}$.

The present study showed that children with ADHD had higher $n-3: n-6$ ratio, $n-3:$ total PUFA ratio and lower total LC $n-3: n-3$ ratio, $n-6: n-3$ ratio compared with age-matched children without ADHD. The reason for this is not clear, but this may partially be explained by the higher $18: 3 n-3$ intake. The present study showed no correlations between any fatty acids including $20: 5 n-3$ and $22: 6 n-3$ and symptoms of ADHD (Table 6). However, positive clinical trials have shown some advantages over placebo for supplementing both $20: 5 n-3$ and $22: 6 n-3^{(21-23)}$, as well as some $n-6$ PUFA $^{(38,39)}$ in the treatment of inattention, impaired cognition or mood disorder, but no benefits from supplementation with $22: 6 n-3$ alone ${ }^{(40,41)}$. This suggests that both $20: 5 n-3$ and $22: 6 n-3$ may be necessary for a positive outcome.

The Australian NRV recommends an adequate intake of $70 \mathrm{mg}$ LC $n-3$ PUFA per day for boys and girls aged $9-13$ years $^{(25)}$. The definition of adequate intake is the average daily nutrient intake level based on observed or experimentally determined approximations or estimates of nutrient intake by a group (or groups) of apparently healthy

Table 6. Comparison of daily PUFA intakes with recommendations from Nutrient Reference Values (NRV), National Heart Foundation (NHF) and International Society for the Study of Fatty Acids and Lipids (ISSFAL)

(Median values and interquartile ranges (IQR))

\begin{tabular}{|c|c|c|c|c|c|c|c|c|c|c|}
\hline & \multicolumn{10}{|c|}{ Fatty acid intake (mg/d) } \\
\hline & \multicolumn{3}{|c|}{ Male } & \multirow[b]{3}{*}{$P$} & \multicolumn{3}{|c|}{ Female } & \multirow[b]{3}{*}{$P$} & \multirow[b]{3}{*}{$\mathrm{NHF}^{(26)}$} & \multirow[b]{3}{*}{ ISSFAL $^{(27)}$} \\
\hline & \multicolumn{2}{|c|}{ Age $6-13(n 57)$} & \multirow{2}{*}{$\frac{\text { Age } 9-13}{\mathrm{NRV}^{(25)}(\mathrm{Al})}$} & & \multicolumn{2}{|c|}{ Age $6-13(n 22)$} & \multirow{2}{*}{$\frac{\text { Age } 9-13}{\mathrm{NRV}^{(25)}(\mathrm{Al})}$} & & & \\
\hline & Median & IQR & & & Median & IQR & & & & \\
\hline $18: 2 n-6$ & 8015 & $6028-10923$ & 10000 & 0.007 & 7450 & $6815-9460$ & 8000 & NS & - & - \\
\hline $18: 3 n-3$ & 1058 & $777-1434$ & 1000 & NS & 973 & $768-1744$ & 800 & 0.007 & - & - \\
\hline LC $n$-3 PUFA & 64 & $21-122$ & 70 & NS & 64 & $37-126$ & 70 & NS & $500^{*}$ & $500^{*}$ \\
\hline
\end{tabular}

Al, adequate intake; $18: 2 n-6$, linoleic acid; $18: 3 n-3, \alpha$-linolenic acid; LC $n-3$ PUFA, long-chain $n-3$ PUFA (sum of $20: 5 n-3,22: 5 n-3$ and $22: 6 n-3$ ).

* Sum of $20: 5 n-3$ and $22: 6 n-3$. 
people that are assumed to be adequate ${ }^{(25)}$. This definition refers to 'apparently healthy people'. The current NRV for LC $n-3$ PUFA intake of $70 \mathrm{mg} / \mathrm{d}$ for children aged $9-13$ years is based on the median intake in the population ${ }^{(36,42)}$, and assumes that the population is healthy, yet the incidence of ADHD in Australia is $11 \%$, which suggests our children population is not entirely healthy. There are other recommendations that suggest much higher optimal intakes of LC $n$-3 PUFA. The International Society for the Study of Fatty Acids and Lipids and the National Heart Foundation of Australia recommend an intake of $500 \mathrm{mg} 20: 5 n-3$ and $22: 6 n-3$ per day, based on a current scientific evidence ${ }^{(26,27)}$. It is likely that the NRV recommendation of $70 \mathrm{mg} / \mathrm{d}$ is set too low and is $13 \%$ of the recommendations set by $500 \mathrm{mg} / \mathrm{d}$ by the National Heart Foundation and International Society for the Study of Fatty Acids and Lipids. Therefore, the present study indicates that all Australian children including those with ADHD are consuming far less than optimal amounts of LC $n$-3 PUFA.

Although few clinical intervention studies showed some evidence of alleviation of behavioural problems on ADHD participants with LC PUFA supplementation ${ }^{(21-23)}$, not all studies agreed with this ${ }^{(39-41)}$. These studies supplemented ADHD children with DHA and other LC PUFA precursors, and no improvements in any ADHD symptoms were observed. This finding led to a hypothesis that these children may have impaired fatty acids' metabolism. A preliminary result from Brookes et al. ${ }^{(43)}$ showed that there is an association between fatty acid desaturase genes (FADS2) and ADHD.

It should be noted that there are limitations associated with self-reported food intake. There was some estimation of serving sizes of foods, e.g. the average child meat serving size of $65 \mathrm{~g}$ was used when the quantity was not specified; the food nutrient databases used do not contain all of the exact foods; hence, substitutions were necessary, e.g. roma tomato was substituted with raw tomato. Another limitation of the present study is the lack of blood analysis as dietary intakes do not necessarily correlate with PUFA levels as shown by Colter et al. ${ }^{(35)}$; however $20: 5 n-3$ and $22: 6 n-3$ do correlate well with circulating blood levels ${ }^{(44)}$.

In summary, the present study found that children with ADHD consumed significantly less meat/egg and fish/ seafood compared to the general population the same age. However, this did not translate to a statistical significantly lower consumption of LC $n-3$ PUFA. The only differences in dietary PUFA intake were lower $20: 4 n-6$ and higher $18: 3 n-3$ intakes. The median $20: 5 n-3$ and $22: 6 n-3$ intakes were adequate when compared with the NRV, but were only $13 \%$ of those recommendations by National Heart Foundation and International Society for the Study of Fatty Acids and Lipids. Given the overall current evidence for the role of LC $n-3$ PUFA in ADHD symptoms and the lower consumption of foods rich in LC $n-3$ PUFA, children with ADHD are encouraged to consume more LC $n-3$ PUFA containing foods for mental health benefits.

\section{Acknowledgements}

The authors gratefully acknowledge the assistance from Karen Walton, Kelly Lambert, Serina Faraji, Janet Bryan and all the children and their parents who participated in the present study. The authors would like to acknowledge the University of Wollongong for funding the study. B. J. M and N. S. initiated and designed the study. N. S. collected the data. K.-H. N. G. and L. R. analysed the data supervised by B. J. $\mathrm{M}$ and N. S. K-.H. N. G. prepared the manuscript and B. J. M., N. S. and L. R. reviewed the manuscript. There was no potential conflict of interest for any authors of the present paper.

\section{References}

1. Sawyer MG, Arney FM, Baghurst PA, et al. (2001) The mental health of young people in Australia: key findings from the child and adolescent component of the national survey of mental health and well-being. Aust N Z J Psychiatry 35, 806-814.

2. American Psychiatric Association (1994) Diagnostic and Statistical Manual of Mental Disorders, 4th ed. Washington, DC: American Psychiatric Association.

3. Richardson AJ \& Puri BK (2000) The potential role of fatty acids in attention-deficit/hyperactivity disorder. Prostaglandins Leukot Essent Fatty Acids 63, 79-87.

4. Richardson AJ (2006) Omega-3 fatty acids in ADHD and related neurodevelopmental disorders. Int Rev Psychiatry 18, $155-172$.

5. Doyle AE, Faraone SV, DuPre EP, et al. (2001) Separating attention deficit hyperactivity disorder and learning disabilities in girls: a familial risk analysis. Am $J$ Psychiatry 158, 1666-1672.

6. Costello EJ, Mustillo S, Erkanli A, et al. (2003) Prevalence and development of psychiatric disorders in childhood and adolescence. Arch Gen Psychiatry 60, 837-844.

7. Barbaresi WJ, Katusic SK, Colligan RC, et al. (2002) How common is attention-deficit/hyperactivity disorder? Incidence in a popultion-based birth cohort in Rochester, Minn. Arch Pediatr Adolesc Med 156, 217-224.

8. Root RWI \& Resnick RJ (2003) An update on the diagnosis and treatment of attention-deficit/hyperactivity disorder in children. Prof Psychol Res Pract 2003, 34-41.

9. Thapar A, O'Donovan M \& Owen MJ (2005) The genetics of attention deficit hyperactivity disorder. Hum Mol Genet 14, $\mathrm{R} 275-\mathrm{R} 282$

10. Milberger S, Biederman J, Faraone SV, et al. (1996) Is maternal smoking during pregnancy a risk factor for attention deficit hyperactivity disorder in children? Am $J$ Psychiatry 153, $1138-1142$.

11. Richardson AJ (2004) Long-chain polyunsaturated fatty acids in childhood developmental and psychiatric disorders. Lipids 39, $1215-1222$.

12. Holman RT (1998) The slow discovery of the importance of omega 3 essential fatty acids in human health. J Nutr 128, $427 \mathrm{~S}-433 \mathrm{~S}$

13. Li D, Sinclair A, Wilson A, et al. (1999) Effect of dietary alphalinolenic acid on thrombotic risk factors in vegetarian men. Am J Clin Nutr 69, 872-882.

14. Burdge G (2004) Alpha-linolenic acid metabolism in men and women: nutritional and biological implications. Curr Opin Clin Nutr Metab Care 7, 137-144.

15. Haag M (2003) Essential fatty acids and the brain. Can J Psychiatry 48, 195-203.

16. Assisi A, Banzi R, Buonocore C, et al. (2006) Fish oil and mental health: the role of $n-3$ long-chain polyunsaturated fatty acids in cognitive development and neurological disorders. Int Clin Psychoparmacol 21, 319-336. 
17. Young $\mathrm{G} \&$ Conquer $\mathrm{J}$ (2005) Omega-3 fatty acids and neuropsychiatric disorders. Reprod Nutr Dev 45, 1-28.

18. Lauritzen L, Hansen HS, Jùrgensen MH, et al. (2001) The essentiality of long chain $n-3$ fatty acids in relation to development and function of the brain and retina. Prog Lipid Res $\mathbf{4 0}$, $1-94$.

19. Stevens LJ, Zentall SS, Deck JL, et al. (1995) Essential fatty acid metabolism in boys with attention-deficit hyperactivity disorder. Am J Clin Nutr 62, 761-768.

20. Mitchell EA, Aman MG, Turbott SH, et al. (1987) Clinical characteristics and serum essential fatty acid levels in hyperactive children. Clin Pediatr 26, 406-411.

21. Sinn N \& Bryan J (2007) Effect of supplementation with polyunsaturated fatty acids and micronutrients on learning and behavior problems associated with child ADHD. J Dev Behav Pediatr 28, 82-91.

22. Richardson AJ \& Montgomery P (2005) The Oxford-Durham study: a randomized controlled trial of dietary supplementation with fatty acids in children with developmental coordination disorder. Pediatrics 115, 1360-1366.

23. Johnson M, Östlund S, Fransson G, et al. (2008) Omega-3/ omega- 6 fatty acids for attention deficit hyperactivity disorder. A randomized placebo-controlled trial in children and adolescents. J Atten Disord 12, 394-401.

24. McLennan W \& Podger A (1997) National Nutrition Survey Selected Highlights Australia 1995. Canberra: Australia Bureau of Statistics, Commonwealth of Australia.

25. National Health and Medical Research Council (2006) Nutrient Reference Values for Australia and New Zealand Executive Summary. Canberra: Commonwealth of Australia.

26. Colquhoun D, Ferreira-Jardin A, Udell T, et al. (2008) http:// www.heartfoundation.org.au/document/NHF/HW_FS_FishOil_ RevEv_FINAL.pdf (accessed September 2008).

27. International Society for the Study of Fatty Acids and Lipids (2004) $\mathrm{http}: / / \mathrm{www}$.issfal.org.uk/index.php?option $=$ com_content\&task $=$ view $\&$ id $=23 \&$ Itemid $=8$ (accessed October 2007).

28. Conners CK (2000) Conners Rating Scales-Revised: Technical Manual. New York: Multi-Health Systems, Inc.

29. Black AE (2000) The sensitivity and specificity of the Goldberg cut-off for EI:BMR for identifying diet reports of poor validity. Eur J Clin Nutr 54, 395-404.

30. Schofield WN (1985) Predicting basal metabolic rate, new standards and review of previous work. Hum Nutr Clin Nutr 39C, Suppl. 1, 5-41.

31. Sullivan BL, Brown J, Williams PG, et al. (2008) Dietary validation of a new Australian food-frequency questionnaire that estimates long-chain $n$-3 polyunsaturated fatty acids. $\mathrm{Br} \mathrm{J}$ Nutr 99, 660-666.

32. Mann NJ, Sinclair AJ, Percival P, et al. (2003) Development of a database of fatty acids in Australian foods. Nutr Dietetics $\mathbf{6 0}$, $42-45$.

33. Department of Health and Ageing (1998) The Australian Guide to Healthy Eating. Canberra: Commonwealth of Australia.

34. Chen JR, Hsu SF, Hsu CD, et al. (2004) Dietary patterns and blood fatty acid composition in children with attention-deficit hyperactivity disorder in Taiwan. J Nutri Biochem 15, 467-472.

35. Colter AL, Cutler C \& Meckling K (2008) Fatty acid status and behavioural symptoms of Attention Deficit Hyperactivity Disorder in adolescents: a case-control study. Nutr $J$ 7, 8-18.

36. Meyer BJ, Mann NJ, Lewis JL, et al. (2003) Dietary intakes and food sources of omega- 6 and omega- 3 polyunsaturated fatty acids. Lipids 38, 391-398.

37. Burgess JR, Stevens L, Zhang W, et al. (2000) Long-chain polyunsaturated fatty acids in children with attention-deficit hyperactivity disorder. Am J Clin Nutr 71, 327S-3230.

38. Richardson AJ \& Puri BK (2002) A randomized double-blind, placebo-controlled study of the effects of supplementation with highly unsaturated fatty acids on ADHD-related symptoms in children with specific learning difficulties. Prog Neuropsychopharmacol Biol Psychiatry 26, 233-239.

39. Stevens L, Zhang W, Peck L, et al. (2003) EFA supplementation in children with inattention, hyperactivity, and other disruptive behaviors. Lipids 38, 1007-1021.

40. Voigt RG, Llorente AM, Jensen CL, et al. (2001) A randomized, double-blind, placebo-controlled trial of docosahexaenoic acid supplementation in children with attention-deficit/hyperactivity disorder. J Pediatr 139, 189-196.

41. Hirayama S, Hamazaki T \& Terasawa K (2004) Effect of docosahexaenoic acid-containing food administration on symptoms of attention-deficit/hyperactivity disorder - a placebocontrolled double-blind study. Eur J Clin Nutr 58, 467-473.

42. Howe PRC, Meyer BJ, Record S, et al. (2006) Dietary intake of long chain omega-3 polyunsaturated fatty acids: contribution of meat sources. Nutrition 22, 47-53.

43. Brookes KJ, Chen W, Xu X, et al. (2006) Association of fatty acid desaturase genes with attention-deficit/hyperactivity disorder. Biol Psychiatry 60, 1053-1061.

44. Sullivan BL, Williams PG \& Meyer BJ (2006) Biomarker validation of a new food frequency questionnaire that estimates long-chain omega-3 polyunsaturated fatty acids. Lipids $\mathbf{4 1}$, $845-850$. 